Cahiers $d u$ MONDE RUSSE

\section{Cahiers du monde russe}

Russie - Empire russe - Union soviétique et États indépendants

$41 / 4 \mid 2000$

Aperçus sur le monde juif

\title{
1953 : la déportation des juifs soviétiques était-elle programmée?
}

\section{Samson Madievski}

\section{OpenEdition}

\section{Journals}

Édition électronique

URL : https://journals.openedition.org/monderusse/59

DOI : 10.4000/monderusse.59

ISSN : $1777-5388$

\section{Éditeur}

Éditions de l'EHESS

\section{Édition imprimée}

Date de publication : 1 octobre 2000

Pagination : $561-568$

ISBN : 2-7132-1383-5

ISSN : $1252-6576$

Référence électronique

Samson Madievski, «1953: la déportation des juifs soviétiques était-elle programmée ? 》, Cahiers du monde russe [En ligne], 41/4 | 2000, mis en ligne le 15 janvier 2007, consulté le 06 septembre 2022

URL : http://journals.openedition.org/monderusse/59; DOI : https://doi.org/10.4000/monderusse.59 


\section{3 : LA DÉPORTATION DES JUIFS SOVIÉTIQUES ÉTAIT-ELLE PROGRAMMÉE?}

À PARTIR DU MILIEU DES ANNÉES 30, alors que se ternit en URSS l'idéologie communiste et que se renforce le régime du pouvoir personnel, Stalin s'appuie de plus en plus en politique sur le nationalisme grand-russe dont l'antisémitisme est un composant traditionnel comme on le sait (et nullement étranger d'ailleurs au dictateur lui-même). Cependant, la conjoncture politique intérieure et extérieure, et, en particulier, l'opposition idéologique et politique au national-socialisme allemand excluait alors toute possibilité de manifestation déclarée d'antisémitisme. Cela était d'autant plus vrai durant la période 1941-1945 lorsque l'Union Soviétique et les démocraties occidentales luttaient contre l'Allemagne hitlérienne (bien que, déjà pendant la guerre, des juifs eussent été secrètement évincés de nombreux postes de direction). Après 1945, dans le contexte de confrontation croissante avec l'Occident (la «guerre froide ») et de durcissement du régime en politique intérieure, la discrimination et la persécution des juifs se renforcent d'année en année.

Il y a un demi-siècle, en 1949, une vague mais sinistre rumeur a commencé à se répandre sur la préparation en URSS d'une déportation des juifs. C'était peu après le saccage du Comité juif antifasciste (EAK - Evrejskij antifašistskij komitet), les arrestations massives des représentants de l'intelligentsia juive et la campagne contre les « cosmopolites » qui s'est étendue à tout le pays. Depuis lors, en Occident, et à partir des années 1990 en Russie, quantité de publications ont paru, abordant ce thème d'une façon ou d'une autre. La plupart d'entre elles considèrent que la déportation des juifs russes dans les lointaines régions de Sibérie et d'ExtrêmeOrient était une action programmée et minutieusement préparée dont la réalisation n'a été empêchée qu'au dernier moment par la mort soudaine de Stalin, son initiateur. La version la plus détaillée en est exposée dans les livres du journaliste Zinovij Šejnis, Grozila deportacii (Menace de déportation) (Moscou, 1991), et Provokacija veka (La provocation du siècle) (Moscou, 1994), dans l'ouvrage du juriste Jakov Ajzenštat, O podgatovke Stalinym genocida sovetskih evreev (La préparation du 
génocide des juifs soviétiques par Stalin) (Jérusalem, 1994), et dans celui du publiciste Fedor Ljass, Poslednij političeskij process Stalina ili nesostojavšijsja genocid (Le dernier procès politique de Stalin ou le génocide qui n'a pas eu lieu) (Jérusalem, 1995).

Voici les éléments qui fondent cette version. À la fin des années 40 , au début des années 50, la décision fut prise, au plus haut niveau, de déporter les juifs soviétiques. Une commission placée sous les ordres personnels de Stalin fut spécialement créée pour la déportation. La présidence en fut confiée à M. A. Suslov, le secrétariat - à N. N. Poljakov, collaborateur des services de sécurité de l'État puis du Comité Central du parti. Pour installer les juifs dans le Birobidjan et en d'autres lieux, on construisit à la hâte des ensembles de baraquements du type de ceux que l'on trouve dans les camps, et les territoires sur lesquels ils se trouvaient furent classés en zones interdites et secrètes. Simultanément, à travers tout le pays, on dressa des listes de personnes de nationalité juive (par l'intermédiaire des services du personnel sur les lieux de travail et par l'administration de l'habitat sur les lieux de vie). Deux listes distinctes furent établies - celle des sangs purs et celle des sangs mêlés et cela dans la mesure où l'opération était prévue en deux étapes.

La déportation devait avoir lieu dans la deuxième quinzaine de février 1953 mais, en raison du retard pris dans la constitution des listes, elle fut reportée à la deuxième quinzaine de mars. La date du jugement des «médecins-comploteurs » fut fixée par Stalin aux 5-7 mars, le châtiment public - aux 11-12 mars. Après quoi, dans toutes les grandes entreprises industrielles du pays, il était prévu de tenir des meetings monstres afin de châtier tous les complices des « assassins en blouses blanches ». L'hystérie antisémite, à son apogée, devait aboutir à des pogromes « spontanés », à des massacres « populaires » des juifs à travers tout le pays. Alors, selon le dessein de Stalin, une lettre ouverte devait lui être adressée par des juifs soviétiques éminents condamnant les «médecins-assassins », ces monstres du genre humain, et demandant que les juifs soient évacués dans des régions reculées du pays afin de les protéger contre de possibles actes de violence perpétrés par leur entourage.

Le ministre des Forces armées de l'URSS, N. A. Bulganin, reçut de Stalin l'ordre d'acheminer vers la capitale ainsi que vers d'autres grandes villes quelques centaines de convois militaires ferroviaires. Des wagons sans châlits étaient prêts sur les voies ferrées proches de Moscou, dans la région de Tachkent ainsi qu'en beaucoup d'autres endroits encore.

Conformément au scénario validé par Stalin, à peine la moitié des déportés devaient atteindre leur lieu de destination. Le long de l'itinéraire des convois, on avait prévu d'organiser des déraillements et des attaques par des « vengeurs du peuple » contre les déportés.

Toute une argumentation théorique marxiste des actions programmées avait été prévue. Stalin avait chargé D. I. Česnokov, rédacteur en chef de la revue Voprosy filosofii, de préparer un travail « scientifique » sur le sujet. Début février 1953, un livre intitulé Počemu neobhodimo vyselit' evreev iz promyšlennyh rajonov strany (Pourquoi il est indispensable d'expulser les juifs des régions industrielles) était 
achevé, approuvé par Stalin et tiré par les éditions du ministère des Affaires intérieures (MVD) d'URSS à un million d'exemplaires. Ce tirage était entreposé dans les locaux du ministère de la Sécurité nationale (MGB) et, le jour J, il devait être diffusé dans tout le pays. Des comptes rendus de cet ouvrage étaient préparés pour les grands journaux, la radio et la télévision.

Sur quelles sources cette version des faits est-elle fondée? Avant tout, sur les déclarations de N. N. Poljakov consignées par deux témoins peu avant sa mort. Ces déclarations sont citées dans le livre de Z. Šejnis, Provokacija veka. La seconde source est celle du témoignage de N.A. Bulganin dans un entretien avec I. I. Etinger, docteur en histoire (voir le recueil : Hronika «dela vračej » (Chronique de l'« affaire des médecins »), pp. 4-7). La troisième source est celle du récit de I. Erenburg à l'écrivain I. Borev à propos d'un entretien avec Hruščev rapportant une prétendue conversation avec Stalin sur une déportation imminente (cf. I. Borev Staliniada (Stalinade) (Moscou, 1990). La dernière source est constituée par les informations citées par Z. Šejnis et I. Borev sur la préparation du livre de D. I. Česnokov (sans indication particulière de leur provenance).

Il faut dire que la version présentée a, dès le début, suscité des interrogations. Par exemple : où peut-on trouver les déclarations de N. N. Poljakov ? Personne ne peut répondre à cette question - en effet ni l'auteur des déclarations ni celui qui les a publiées le premier ne sont aujourd'hui vivants. De plus, si Hruščev a bien parlé à Erenburg d'une déportation imminente, pourquoi n'en a-t-il pas fait mention dans ses mémoires ( Vospominanija, New York, 1979) où, plus d'une fois, il est fait allusion à l'antisémitisme de Stalin? Enfin, plus important encore : si la commission pour la déportation a bien réellement existé, il devrait en rester des traces documentaires. Où sont-elles ? Et en tout dernier lieu, si le livre de Česnokov a bien eu le grand tirage indiqué, est-il possible qu'aucun exemplaire ne se soit conservé ? Et même si la totalité du tirage a été détruite, il devrait rester des témoins - ouvriers de la composition, typographes, correcteurs... Où sont-ils donc ?

En 1994, est paru à Moscou un volumineux ouvrage (400 pages) signé de l'historien russe G. Kostyrčenko, V plenu u krasnogo faraona. Političeskie presledovanija evreev $v$ SSSR v poslednee stalinskoe desjatiletie (Prisonniers du pharaon rouge : les répressions politiques contre les juifs en URSS dans la dernière décennie du règne de Stalin, trad. française : Arles, 1997). Le livre porte comme sous-titre : «Enquête documentaire ». Effectivement, à la différence de tous ceux qui auparavant ont écrit sur ce sujet, G. Kostyrčenko a extrait un important fonds documentaire des archives ultra-secrètes du Comité Central du parti et du KGB d'URSS. Ces documents se trouvent désormais dans le Centre de conservation et d'étude des documents sur l'histoire contemporaine de Russie (RCHIDNI devenu RGASPI, Archives nationales de Russie d'histoire sociale et politique), dans les Archives d'État de la Fédération de Russie (GARF), dans les Archives centrales des Services fédéraux de contre-renseignements et dans les Archives du Président.

L'auteur n'a pas découvert dans ces fonds d'archives la confirmation par les documents de la thèse de la préparation d'une déportation des juifs soviétiques. Il l'a donc alors qualifiée d'hypothèse que les enquêtes à venir devront soit confirmer soit réfuter. 
En 1998, un symposium sur le thème de « La dernière période du stalinisme et la question juive » s'est tenu en Allemagne; il a réuni des spécialistes renommés qui travaillent sur cette question et qui sont venus de Russie, d'Israël, de la République Fédérale d'Allemagne, de la République tchèque, de Pologne et de Hongrie. Le symposium était co-organisé par l'Institut d'études des pays d'Europe Centrale et Orientale et par la Chaire d'histoire contemporaine des pays de la région de l'Université catholique d'Eichstätt. La Russie était représentée par Gennadij Vasil'evič Kostyrčenko (de l'Institut d'histoire de la Russie de l'Académie des sciences) et par le professeur Vladimir Pavlovič Naumov, docteur en histoire (secrétaire de la Commission pour la réhabilitation des victimes des répressions politiques près du président de la Fédération de Russie). Leur « duel » a constitué, à nos yeux, l'événement central du symposium.

Kostyrčenko a repris son argument de base : nous ne disposons pas, à ce jour, de documents officiels prouvant la préparation d'une déportation. Cependant, à supposer que ces documents aient bien existé, ils auraient immanquablement défrayé la chronique quelque secrets qu'ils aient pu être. Ce point de vue est fondé sur bien des raisons. Rappelons-nous : on sait bien que n'ont pas été détruits des documents aussi odieux et dangereux pour la réputation du communisme soviétique que le procès-verbal secret du Pacte de non-agression germano-soviétique daté du 28 septembre 1939, incluant la carte du partage de la Pologne, pas plus que le procès-verbal de la session du Politbjuro du Comité Central du parti (VKP(b)) en date du 5 mars 1940 chargeant le NKVD de liquider les officiers polonais retenus prisonniers dans les camps soviétiques. Pendant de nombreuses années, on a officiellement nié l'existence de ces documents mais, après la chute du communisme en 1991, on les a retrouvés dans les Archives de président parmi des papiers classés « Dossier spécial » (Osobaja papka).

L'autre argument de G. Kostyrčenko, selon lequel des fonctionnaires aussi avertis de la cuisine politique de Stalin que l'étaient L. M. Kaganovič et P. A. Sudoplatov déclarent ne jamais avoir entendu parler d'un tel plan, ne nous semble pas tellement convaincant. Kaganovič qui passe habituellement sous silence, nie ou couvre les crimes de Stalin s'est, en l'occurrence, exprimé en ces termes : «Il n’y a pas eu de discussion sur ce thème en ma présence » - tout en soulignant qu'il ne faisait alors plus partie du cercle des plus proches de Stalin (cf. le livre de F. Čuev, Tak govoril Kaganovič. Ispoved' stalinskogo apostola (Ainsi parlait Kaganovič. Confession d'un disciple de Stalin) (Moscou, 1992, pp. 41, 64, 175). Ce qui vient d'être dit concerne d'autant plus Sudoplatov. Pendant la période 1951-1953, à la tête du «Bureau spécial » du MGB pour les renseignements et actes de diversion, dont la mission était de lutter contre les « ennemis de l'extérieur », il pouvait tout à fait ne pas être au courant de ce qui se tramait au sommet dans la section de lutte contre les « ennemis de l'intérieur ». Quant à A I. Mikojan, comme l'ont montré ses mémoires récemment publiés, il estimait qu' « on préparait l'expulsion des juifs hors de Moscou, de manière volontairement coercitive ». (A. I. Mikojan, Tak bylo. Razmyšlenija o minuvšem (C'était ainsi. Réflexions sur le passé)) (Moscou, 1999, pp. 535 -536). 
Les autres arguments de l'auteur paraissent encore moins convaincants. En particulier celui selon lequel on manquait d'instructions venant du sommet de l'État pour la mise en œuvre de la déportation, ou celui selon lequel il aurait fallu au préalable modifier la législation en vigueur de manière à rendre légal l'antisémitisme comme cela avait été fait par l'Allemagne hitlérienne de 1933 à 1941. De surcroît, il aurait fallu changer l'idéologie officielle « qui, en dépit de la pression chauvine du stalinisme, conservait encore le romantisme de l'internationalisme bolchevik, idéologie à laquelle étaient étrangers la discrimination nationale et plus encore le racisme». Et pour mettre en pratique des changements aussi profonds à une échelle aussi vaste, il fallait du temps or Stalin n'en avait pas. En outre, «pratiquement tous les plus proches compagnons d'armes du chef surveillaient avec une tension croissante les manifestations de ses phobies juives redoutant, non sans raison, que tout cela se transforme en règlements de compte à leur égard ».

Que peut-on dire à cela ? L'internationalisme apparent de l'idéologie communiste n'a pas, on le sait, constitué un obstacle à la déportation de quatorze (!) peuples soviétiques; et il n'y a pas eu besoin pour cela d'introduire des changements dans la législation en vigueur. Les déportations étaient motivées politiquement pour des raisons de sécurité, etc. Il ne fait aucun doute que, concernant la déportation des juifs soviétiques, on aurait trouvé des arguments « de poids » : leur hostilité à la cause du socialisme (c'est précisément ainsi que, selon les dires de I. Borev, l'expulsion était justifiée dans le livre de Česnokov); ou le fait que « tous les juifs sont des nationalistes, des agents des services de renseignements américains » (ainsi s'exprimait Stalin à la séance du bureau du présidium du Comité Central du parti le 1er décembre 1952, d'après la note du vice-président du Conseil des ministres de l'URSS, V. A. Malyšev).

Quant à la préparation psychologique d'actions à grande échelle contre les juifs, elle fut entreprise au moins à partir de 1949 (par la campagne contre les « cosmopolites »). L'hystérie propagandiste qui s'est déchaînée avec « l'affaire des médecins », a conduit, selon G. Kostyrčenko, à « une explosion d'antisémitisme populaire » dans laquelle l'agressivité et le désir de régler leurs comptes aux « assassins en blouses blanches » se sont mêlés à la peur panique et viscérale qu'ils inspiraient. Nombreux furent ceux qui reportèrent ces sentiments sur l'ensemble des juifs (en vertu du principe selon lequel « ils sont tous pareils »). On avait ainsi la base psychologique nécessaire à l'adoption massive, « nationale », de mesures punitives appliquées à grande échelle.

Cependant, G. Kostyrčenko pense que l'attitude désapprobatrice de ses proches et la violente réaction de l'Occident à « l'affaire des médecins » ont contraint Stalin à reconnaître « qu'il entraînait le pays dans une impasse idéologique et politique », et à commencer à chercher une issue à cette situation. Des signes de cette démarche sont perceptibles pour l'historien russe dans l'atténuation, après le 20 février 1953, de la campagne de propagande autour de «l'affaire des médecins » et dans la préparation par l'Agitprop du Comité Central de la lettre destinée à Stalin au nom des juifs soviétiques les plus connus. 
Il se trouve que le texte de cette lettre, publiée pour la première fois par la revue Istočnik $(1,1997)$, ne comporte pas de demande de déplacement des juifs. Il est dit dans cette lettre que, en dépit des efforts déployés par les ennemis « pour étouffer chez les juifs la conscience du devoir civique des citoyens soviétiques, [...] pour transformer les juifs de Russie en espions et en ennemis du peuple russe et par là même pour préparer le terrain à la réactivation de l'antisémitisme, cette séquelle terrible du passé, $[\ldots]$ le peuple russe comprend que l'immense majorité de la population juive d'URSS est bien l'amie du peuple russe ». La lettre s'achevait de manière tout à fait inattendue par une demande d'autorisation de publier un journal destiné à « de larges couches de la population juive d'URSS et de l'étranger ».

Il est vrai que cette lettre comportait une condamnation sévère de tous les ennemis officiels d'alors : « les médecins-assassins, l'impérialisme américain, le sionisme international, l'État d'Israël avec lequel la Russie avait rompu ses relations diplomatiques. Cependant, on ne peut qu'être d'accord avec G. Kostyrčenko : les phrases rapportées précédemment se démarquent bien du contenu et du ton de la propagande antérieure. En tout cas, ce texte ne va pas dans le sens de la version selon laquelle une déportation des juifs soviétiques devait avoir lieu deux jours, deux semaines ou deux mois plus tard. En conséquence, les raisons pour lesquelles I. Erenbourg et un certain nombre d'autres personnalités ont refusé de le signer sont différentes de celles que les partisans de la version indiquée avançaient (on estimait que les premiers refusaient de demander « une émigration de plein gré »). Quant à Erenburg, on peut considérer sa position établie par la publication de son appel à Stalin dans ce même numéro de la revue Istočnik.

Voici comment on comprend généralement la conclusion de G. Kostyrčenko sur la question qui nous intéresse. Durant la période 1949-1953 « un sentiment tragique de désolation et de désespoir, provoqué par l'atmosphère d'antisémitisme croissant, se fait jour dans le milieu juif. Ce sentiment était, dans une certaine mesure, favorisé par la mentalité traditionnelle juive qui s'est formée au cours d'une expérience millénaire de persécutions, d'appréhension permanente d'une catastrophe nationale. Et le souvenir récent du bannissement de peuples entiers, accusés de collaboration avec l'ennemi pendant la guerre, y contribuait aussi. » Il n'est donc pas étonnant que « la conscience terrorisée de la population juive fût imprégnée des plus sombres pressentiments », allant même jusqu'à attendre l'exil généralisé en Sibérie. Et cela, même si, comme le suppose l'auteur, aucun plan de ce genre n'existait au sein du pouvoir à ce moment-là.

Le professeur V. Naumov s'oppose à G. Kostyrčenko avant tout sur un point essentiel - l'absence de documents confirmant la préparation de la déportation. Certes, dit-il, nous ne disposons pas aujourd'hui de ces documents. Mais il se peut encore que nous les trouvions. Nous sommes encore loin d'avoir étudié en totalité le fonds des Archives du président et les documents de l'ex-KGB. Et quand bien même nous ne retrouverions pas ces documents, cela ne prouve pas qu'ils n'ont pas existé puisqu'on aurait très bien pu les détruire. On n'aurait pas pratiqué de destruction de documents? Que répondre à cela ! Il est prouvé par exemple que, à l'automne 1954, nombre de documents ont été retirés des archives du Comité Central et du Comité de 
Moscou du PCUS ainsi que du Comité Central du parti communiste ukrainien pour être détruits parce qu'ils mettaient en cause N. S. Hruščev (ce sujet fut d'ailleurs abordé en 1957 au plénum de juin du Comité Central).

Quant à la décision de l'instance suprême de déplacer les juifs, on n'en est simplement pas arrivé jusque-là. V. Naumov, au cours de son intervention à Eichstätt a rapporté un fait dont on ne peut nier l'importance. Il se trouve que la déportation de toute une catégorie de peuples du Nord-Caucase a été légalisée post factum par des décrets du Comité national de défense d'URSS (GKO - Gosudarstvennyj komitet oborony) alors que l'opération d'expulsion de centaines de milliers de personnes, accompagnée de l'extermination sur place de tous ceux que l'on ne pouvait emmener, avait en réalité pris fin. Kruglov, Kobulov, Apolonov qui dirigeaient cette opération avec les pleins pouvoirs du GKO agissaient sur la base d'instructions orales.

V. Naumov considère comme établi le fait que les autorités étaient bien en possession de listes de personnes susceptibles d'être déportées à partir de Moscou. Ces listes étaient dressées sur indication du comité municipal (gorodskij) et des comités d'arrondissements (rajonnye) du parti sous la surveillance des organes du MGB. Pour ce qui est des lieux d'installation, Naumov a alors attiré l'attention sur une décision prise en janvier 1953 par le Politbjuro de construire un camp gigantesque pouvant accueillir de 150 à 200000 personnes considérées comme « criminels étrangers particulièrement dangereux ». Or, dans la mesure où les personnes de cette catégorie, détenues en prison et dans les camps, étaient alors en nombre nettement inférieur, on peut s'interroger sur l'identité de la population qui devait remplir ce nouvel îlot du Goulag. Cependant, notons dès maintenant que la capacité d'hébergement envisagée pour le nouveau camp ne correspond pas, peu s'en faut, à la quantité de juifs alors recensés en URSS; en outre, «les peuples condamnés à l'exil »n'étaient pas installés dans des camps mais vivaient parmi les populations de régions, de territoires et de républiques reculés.

V. Naumov écarte l'argument comme quoi les fonctionnaires très haut placés n'étaient pas mis au courant des plans de déportation, rappelant que, à partir de la fin des années 30, Stalin prenait de plus en plus souvent des décisions importantes entièrement seul, sans même en informer ceux qui devaient être affectés directement à l'exécution de la tâche. Un exemple frappant en est le cas du chef d'étatmajor général de l'Armée Rouge (RKKA), le maréchal B. M. Šapošnikov, qui a eu connaissance de l'entrée des troupes russes en Finlande en 1940... par les journaux alors qu'il se trouvait en maison de repos.

Le principal contradicteur, Kostyrčenko, qualifie « d'illusion » l'opinion selon laquelle Stalin n'avait pas la liberté d'agir sur la question de la déportation des juifs à cause de la position de son entourage.

V. Naumov rejette l'affirmation selon laquelle il aurait été impossible d'expulser les juifs en raison de leur dispersion sur tout le territoire de la partie européenne de l'URSS en se référant à un précédent : tous les Tatars et les Grecs de Crimée qui habitaient en dehors des bords de la Mer Noire ont pourtant été retrouvés et ont été réunis à leurs compatriotes. 
Tels sont aujourd'hui les arguments de base et les contre-arguments des deux parties en présence dans ce débat qui perdure. De toute évidence, chacune des deux parties apporte des arguments sérieux et de poids. La question posée dans le titre de cet article demeure à ce jour ouverte. Espérons que les recherches et les enquêtes en cours permettront d'y apporter enfin une seule et même réponse ne suscitant plus ni doute ni débat.

P. S. Avant de présenter ce document à la publication, j'ai téléphoné à V. Naumov et à G. Kostyrčenko pour savoir ce qu'ils pouvaient ajouter aujourd'hui à ce qui s'était dit lors du symposium. V. Naumov a annoncé avoir trouvé dans le témoignage de Rjumin l'indication selon laquelle, en 1952, en accord avec Stalin, il lui avait présenté des propositions de déportation des juifs. G. Kostyrčenko a réagi selon son habitude, en posant une contre-question : et où peut-on les trouver, ces propositions ? Pour sa part, il a fait savoir que l'étude de la documentation provenant de l'administration des chemins de fer de Moscou, couvrant la période févriermars 1953, ne révèle sur ce lieu aucune accumulation inhabituelle de matériel roulant...

(Traduit du russe par Françoise Cordes)

Schurzelter Str. 551

52074 Aachen

e-mail :madievski.averbuh@01019freenet.de 